\title{
Solução exata da equação de Schrödinger para movimento de uma partícula em um campo magnético paramétrico
}

\author{
Exact solution of the Schrödinger equation for the movement of a particle in a parametric magnetic
} field

Solución exacta de la ecuación de Schrödinger para el movimiento de una partícula en un campo magnético paramétrico

\section{Resumo}

Resolvemos de modo exato a equação de Schrödinger para um sistema quadrático dependente do tempo para uma partícula que se movimenta sob a influência de um campo magnético com oscilação paramétrica. Aplicamos o método de desacoplamento, o qual adota uma transformação de coordenadas espaço-temporal de Ray-Reid (Nassar, 1990). A ideia fundamental do problema é obter uma equação tipo partícula livre de Schrödinger. Desse modo, foi possível determinar a função de onda e a densidade de probabilidade da partícula na forma de uma função de vibração paramétrica. Mostramos que as regiões de estabilidades e instabilidades são determinadas pelo espaço de fase definidos pelos parâmetros de controle da equação. Determinamos, como resultado inédito, os valores discretos que o campo magnético pode assumir em termos das funções de Mathieu.

Palavras-chave: Equação de Schrödinger; Sistema quadrático dependente do tempo; Transformação espaçotemporal; Equação e funções de Mathieu; Ressonância e oscilações paramétricas.

\footnotetext{
Abstract

We solved the Schrödinger equation exactly for a time-dependent quadratic system for a particle that moves under the influence of a magnetic field with parametric oscillation. We apply the decoupling method, which adopts a transformation of Ray-Reid's spatio-temporal coordinates (Nassar, 1990). The fundamental idea of the problem is to obtain a Schrödinger free particle equation. In this way, it was possible to determine the wave function and the probability density of the particle in the form of a parametric vibration function. We show that the regions of stability and instability are determined by the phase space defined by the equation's control parameters. We determined, as an unprecedented result, the discrete values that the magnetic field can assume in terms of Mathieu functions.

Keywords: Schrödinger's Equation; Time-dependent quadratic system; Spatio-temporal transformation; Mathieu equation and functions; Resonance and parametric oscillations.
} 


\section{Resumen}

Resolvimos la ecuación de Schrödinger exactamente para un sistema cuadrático dependiente del tiempo para una partícula que se mueve bajo la influencia de un campo magnético con oscilación paramétrica. Aplicamos el método de desacoplamiento, que adopta una transformación de las coordenadas espacio-temporales de Ray-Reid (Nassar, 1990). La idea fundamental del problema es obtener una ecuación de partículas libres de Schrödinger. De esta forma, fue posible determinar la función de onda y la densidad de probabilidad de la partícula en forma de función de vibración paramétrica. Mostramos que las regiones de estabilidad e inestabilidad están determinadas por el espacio de fase definido por los parámetros de control de la ecuación. Determinamos, como resultado sin precedentes, los valores discretos que el campo magnético puede asumir en términos de funciones de Mathieu.

Palabras clave: Ecuación de Schrödinger; Sistema cuadrático dependiente del tiempo; Transformación espaciotemporal; Ecuación y funciones de Mathieu; Resonancia y oscilaciones paramétricas.

\section{Introdução}

Este artigo é dedicado à solução exata analítica da equação de Schrödinger para uma lagrangeana quadrática dependente do tempo. Muitos fenômenos físicos na escala atômica foram elucidados para os casos de sistemas dinâmicos com propriedades lineares. No entanto, a maioria dos fenômenos encontrados na natureza possuem fontes que obedecem a uma dinâmica não-linear, cujos parâmetros são dependentes da posição ou do tempo. Nesses casos, recorremos aos métodos perturbativos ou numéricos (Sudiarta, 2008), que por sinal são muito limitados, deixando a desejar informações mais detalhadas do sistema. Um desses sistemas, que possui grande importância e aplicabilidade em vários ramos da ciência, é o descrito pelas oscilações paramétricas. Há na literatura outros trabalhos relevantes nessa linha para soluções exatas analíticas usando outras geometrias (Schmidt, 2019 e 2020) e extensões para campos elétricos, Jesus et. al. (1999).

A física paramétrica tem sido uma área de intensas pesquisas nas últimas décadas, principalmente em física teórica, matemática e engenharia. É um estudo voltado principalmente ao tratamento das ressonâncias paramétricas de sistemas nãolineares. Essas oscilações são estudadas em três modelos dinâmicos: vibrações paramétricas (Magnus, 1966), vibrações nãolineares com amortecimento linear (equação de Duffing) e as vibrações com amortecimento não-linear (equação Van der Pol). Essas equações são caracterizadas pela dependência temporal de um dos parâmetros de controle, a qual leva a uma variação da amplitude da oscilação.

No caso específico da equação de Hill (Magnus, 1966), temos duas subclasses bastante conhecidas na literatura: as equações de Mathieu (Mathieu, 1868) e as de Lamé (Magnus, 1966). As equações de Mathieu, de que trata este trabalho, um dos parâmetros (parâmetros de controle) é uma função trigonométrica do tipo seno ou cosseno. As soluções, dessas equações apresentam infinitas bandas de ressonância no espaço de fase definido a partir dos seus parâmetros é mostrado no diagrama de Strutt (Nayfeh \& Mook, 1979) e possuem uma expressão fechada para o expoente de Floquet (Floquet, 1883), as equações de Lamé são aquelas em que um dos parâmetros é uma função elíptica de Jacobi (Filho, 1994).

Em regime de pequenas oscilações encontramos diversos sistemas que são descritos pela equação de Mathieu. O parâmetro temporal que controla o seu movimento mostra que, em determinadas condições, as amplitudes das oscilações aumentam rapidamente dizemos, nesse caso, que o sistema está em um regime de instabilidade paramétrica, fora desse regime a amplitude de oscilação mantêm-se constante e o sistema é estável. Essas oscilações são bastante comuns em casos simples de física-matemática, como de um pêndulo de comprimento variável com o tempo e ponto de suspensão com movimento na direção vertical (Landau, 1971). Em situações com maior complexibilidade encontramos, por exemplo: a produção de partículas na presença de campos eletromagnéticos fortes (Grib, 1994); em processos de polarização de meios ópticos (fibras ópticas); em circuitos RLC os efeitos não-lineares ocorrem devido a variação periódicas da capacitância, levando ao aparecimento dos amplificadores paramétricos; em física de partículas e campos os fenômenos de ressonância paramétrica funcionam como um mecanismo de produção de matéria (Zanchin, 1998); em Condensados de Bose-Einstein ocorre ressonância paramétrica nas distâncias relativas entre dois sólitons (Barros, 2005). Diante das diversas evidências e 
aplicabilidades desse movimento em várias áreas da ciência, apresentamos um estudo nesta direção. Neste artigo calculamos o Propagador de Feynman da equação de Schrödinger para uma partícula em um campo magnético paramétrico tipo função de Mathieu; esse problema generaliza o problema de Landau para uma partícula em um campo magnético constante (Landau, 1977). Para resolver o problema usamos uma técnica desacoplante envolvendo uma transformação de variáveis envolvendo espaço e tempo - transformação espaço-temporal (Bassalo, 1993).

A metodologia usada neste trabalho será uma pesquisa de origem teórica e qualitativa (Demo, 2000), pois iremos aprimorar fundamentos teóricos relevantes no âmbito da mecânica quântica via equação de Schrödinger, tentando compreender fenômenos ligados ao movimento de partículas sujeitas a campos magnéticos. Outra maneira de abordar esse conteúdo de forma investigativa no ensino de Física, principalmente voltada para o ensino médio e graduação poderia ser elaborada como Silva et. al (2020).

\section{Movimento para um Campo Magnético Paramétrico Externo}

Considere o operador hamiltoniano de uma partícula na presença de um campo magnético

$\hat{H}=\frac{1}{2 m}\left[\left(\widehat{P}+\frac{e}{e} A\right)^{2}\right], \quad \hat{P}=\hat{p}_{x} i+\hat{p}_{y} j+\hat{p}_{k} k$

Escolhendo o potencial vetor derivado da intensidade do campo magnético e tomando a direção do eixo-z, para o campo magnético. Tal que $\left(A_{y}=A_{z}=0\right)$, então,

$A_{x}(t, y)=-\mathscr{H}(t) y$,

Considere a intensidade do campo magnético na forma paramétrica

$\mathscr{H}(t)=\mathscr{H}_{0} \alpha(t), \quad \alpha(t)^{2}=1-4 g^{2} \cos \omega t$

O operador hamiltoniano do sistema equação (1) é dado explicitamente usando equações (2) e (3) em (1).

$\hat{H}(p, t)=\frac{1}{2 m}\left[\hat{p}_{x}^{2}-\frac{e}{e} \mathscr{H}_{0} \alpha(t) y\right]^{2}+\frac{1}{2 m}\left(\hat{p}_{y}^{2}+\hat{p}_{z}^{2}\right)$,

este operador não contém as coordenadas x e z explicitamente, portanto, as componentes do momento nessas direções comutam com $\hat{H}$, isto é, $\hat{p}_{x}$ e $\hat{p}_{z}$ são conservados. A equação de onda $\hat{H} \Psi=i \hbar \frac{\partial}{\partial t} \Psi$ correspondente a hamiltoniana equação (4) é dada por:

$\left\{\frac{1}{2 m} \hat{p}_{x}^{2}+\frac{1}{2 m}\left(\frac{e}{c} \mathcal{H}_{0}\right)^{2} \alpha(t)^{2} y^{2}+\frac{e}{m e} \mathcal{H}_{0} \alpha(t) y \hat{p}_{x}+\frac{1}{2 m}\left(\hat{p}_{y}^{2}+\hat{p}_{z}^{2}\right)\right\} \Psi=\operatorname{i\hbar } \frac{\partial}{\partial t} \Psi$

A relação de comutação entre o operador diferencial momento e posição impõem a condição,

$\hat{p}_{i} x_{k}-x_{k} \hat{p_{i}}=-i \hbar \delta_{i k} ;(i, k=x, y, z)$ 
Tal que a equação (5) tome a forma,

$\operatorname{in} \frac{\partial}{\partial t} \Psi=\frac{1}{2 m}\left\{\hat{p}_{x}^{2}+\left(\frac{e}{e} \mathcal{H}_{0}\right)^{2} \alpha(t)^{2} y^{2}+\frac{2 e}{m e} \mathcal{H}_{0} \alpha(t) \hat{p}_{x} y+\left(\hat{p}_{y}^{2}+\hat{p}_{z}^{2}\right)\right\} \Psi$

Para resolver a equação acima, vamos considerar a transformação polar complexa,

$\Psi\left(x_{x}, z_{z}, t\right)=\exp \left[\frac{i}{n}\left(p_{x} x+p_{z} z\right)\right] \Phi(y, t)$

Após uma manipulação algébrica obtemos,

$i \hbar \frac{\partial}{\partial t} \Phi(y, t)=\frac{1}{2 m} p_{y}^{2} \Phi(y, t)+\frac{m}{2}\left[\beta^{2}(t)\left(Y^{2}+2 y Y+y^{2}\right)+\left(\frac{p_{z}}{m}\right)^{2}\right] \Phi(y, t)$

definindo covenientemente as funções:

$\beta(t)=\left(\frac{e H_{0}}{e}\right) \alpha(t)$

$\mathrm{e}$

$Y(t)=\left(\frac{p_{x} c}{e \%}\right) \alpha(t)$

Para que a equação (9) tome a forma da equação de Schrödinger com hamiltoniano de um sistema quadrático dependente do tempo (Bassalo, 1993) usamos a transformação:

$\Phi(y, t)=\exp [y t] x(y, t)$

Que substituída na equação (09), encontramos,

$\gamma=-\frac{i m}{2 h}\left(\beta^{2} Y^{2}(\mathrm{t})+\frac{p_{z}^{2}}{m^{2}}\right)$

Para determinar a função $\chi(y, t)$ devemos resolver a seguinte uma equação diferencial de Schrödinger do tipo lagrangeano quadrático dependente do tempo (Bassalo, 1993),

$i \hbar \frac{\partial}{\partial t} x(y, t)=-\frac{\hbar^{2}}{2 m} \nabla_{y}^{2} x(y, t)+\left[\frac{1}{2} b(t) y^{2}-c(t) y\right] \chi(y, t)$

sendo: 
$b(t)=\frac{m e H_{0}}{e}\left(1-4 g^{2} \cos \omega t\right), \quad c(t)=\frac{m p_{x} e H_{0}}{e} \sqrt{1-4 g^{2} \cos \omega t}$

Nessas condições a função (11) é reescrita como

$\Phi(y, t)=\exp \left[-\frac{i m}{2 h}\left(\beta^{2} Y^{2}+\frac{p_{z}^{2}}{m^{2}}\right) t\right] \chi(y, t)$

Para determinar a solução da equação (14), lançamos mão do reescalonamento do espaço-temporal (Bassalo, 1993)

$y=s(\tau) \bar{y}+p(\tau)$, com $\quad \frac{d \pi(t)}{d t}=\mu(t)$

Nesse novo sistema de coordenadas, dado pela equação (17) e tomando ( $\hbar=1)$, a equação (14) toma a seguinte forma

$i \mu\left[\frac{\partial}{\partial \tau}+\left(\frac{g y}{g} \bar{y}+\frac{p y}{g}\right) \frac{\partial}{\partial y}\right] \bar{X}(\bar{y}, \tau)=-\frac{1}{2 m s^{2}} \frac{\partial^{2}}{\partial^{2} \bar{y}} \bar{X}+\left[\frac{1}{2} b(t)(s \bar{y}+p)^{2}-c(t)(s \bar{y}+p) \bar{X}\right.$

Usando a transformação WKB (Wentzel, 1981), temos

$\bar{X}(\bar{y}, \tau)=\exp [i f(\bar{y}, \tau)] \Theta(\bar{y}, \tau)$

Desta forma, nesse novo sistema de coordenadas obtemos:

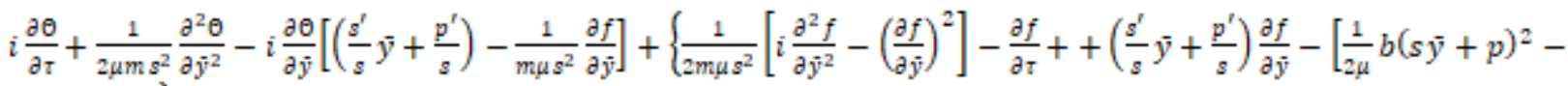
$c(s \bar{y}+p)]\} \Theta(\bar{y}, \tau)=0$

Nas condições desejadas de partícula livre (Bassalo, 1993), obtemos as seguintes condições:

(I) $\quad f(\bar{y}, \tau)=m s \mu p^{s} \bar{y}+\frac{1}{2} s s^{s} m \mu \bar{y}^{2}+\frac{i}{2} \ell_{n} \sqrt{s}+\frac{1}{2} p p^{0}+\frac{1}{2} l(t)$

(II) $\quad \ddot{p}(t)+h_{0}^{2}\left(1-4 g^{2} \cos ^{2} \omega t\right) p(t)=\delta\left(1-4 g^{2} \cos ^{2} \omega t\right)=0$,

$h_{0}^{2}=\frac{m e H_{0}}{e}$ e $\delta^{2}=\frac{e H_{0} p_{x}}{m^{2} \mathrm{c}}$

(III) $\quad s(t)+\omega_{0}^{2}\left[1-\eta \cos ^{2} \omega t\right] s(t)=0, \quad \omega_{0}^{2}=\frac{e \mathcal{H}_{0}}{e}$

Essa é a equação de Mathieu, é uma equação diferencial ordinária, tem grande importância em diversas áreas da física e da matemática, especialmente na análise de comportamento oscilatório e coordenadas elípticas cilíndricas, sendo, $\omega_{0}$ a 
frequência natural de oscilação do sistema, $\omega$ a frequência com a qual o parâmetro oscilante varia e $\left(\eta=4 g^{2}\right)$ a amplitude de variação da frequência natural. A Equação de Mathieu, é uma subclasse das equações de Hill (Magnus, 1966) bastante conhecidas e estudadas e que possuem ligação com o fenômeno de ressonância paramétrica: As equações de Mathieu são definidas pelas funções trigonométricas tipo seno ou cosseno. Essas equações apresentam infinitas bandas de ressonância no espaço de fase definido pela sua frequência natural $\omega_{0}$ e sua amplitude $\eta$.

$$
m \mu(t) s^{2}(t)=M_{0}=\text { cte }
$$

\section{Obtenção da equação de Schrödinger}

A partir dos resultados anteriores podemos escrever a equação de Schödinger como:

$i \hbar \frac{\partial}{\partial \tau} \Theta(\bar{y}, \tau)+\frac{\hbar^{2}}{2 M_{0}} \frac{\partial^{2}}{\partial \tilde{y}^{2}} \Theta(\bar{y}, \tau)=0$

A solução já bem explorada na literatura (Tannoudji, 2006. \& Abramowitz, 1965), é dada pela expressão

$\theta(\bar{y}, \tau)=\sqrt{\frac{m \mu(t) s^{2}(t)}{2 \pi i h x(t)}} \int_{-\infty}^{+\infty} \varphi\left(\bar{y}_{0}\right) \exp \left[\frac{i M_{0}}{2 h \pi(t)}\left(\bar{y}-\bar{y}_{0}\right)\right] d \bar{y}_{0}$

A função de onda solução da partícula num campo magnético paramétrico é, portanto,

$\Psi(x, y, z, t)=\exp \left\{\frac{i}{h}\left[p_{x} x+p_{z} z-\frac{m^{2}}{2 h}\left(\beta^{2} Y^{2}+\frac{k_{z}^{2} z}{m^{2}}\right)\right]\right\} \times \exp [i f(\bar{y}, \tau)] \Theta(\bar{y}, \tau)$

A densidade de probabilidade quântica, é dada pela expressão:

$\rho(x, y, z, t)=|\Theta(\bar{y}, \tau)|^{2}=\left(\frac{1}{2 \pi n h}\right) \frac{1}{s(t)} \int_{t} s^{2}\left(t^{\prime \prime}\right) d t^{\prime}$

Um ponto muito importante na equação (28), está no fato de a densidade de probabilidade $\rho(x, y, z, t)$ apresentar uma periodicidade paramétrica no módulo da função s(t). Fenômenos como esses ocorrem em sistemas não-lineares e são mais fáceis de serem visualizados quando se realiza uma análise paramétrica sobre o sistema. Nesse estudo, geralmente adota-se a amplitude ou a frequência da excitação como parâmetros variáveis, e os demais são mantidos constantes. Nesse procedimento o parâmetro escolhido (parâmetro de controle) é submetido a mudanças suaves, desse modo, encontramos faixas seguras de oscilações em que a excitação ocorre, evitando, assim um comportamento de instabilidade no sistema (caóticos). Ou seja, evitando que o sistema apresente vibrações com amplitude excessiva (ressonância). Por esta razão, é importante uma análise detalhada do comportamento da função $s(t)$ na função que controlar a densidade de probabilidade $\rho(x, y, z, t)$, equação (28). Para este fim tomaremos a forma padrão da equação de Mathieu (Gutiérrez, 2003). 
$\frac{d^{2} S(\phi)}{d \phi^{2}}+\left(\lambda-k^{2} \cos \phi\right) S(\phi)=0$,

onde:

$\lambda=\left(\frac{e H_{i}}{e}\right)^{2} \quad$ e $\quad k=2 g\left(\frac{e H_{\omega}}{e}\right)$

Esta equação tem sido bastante estudada na literatura, as regiões para as quais as soluções são aceitáveis dependem dos valores dados as constantes $\lambda \in k$, estão completamente determinadas para todos os valores dos parâmetros (Nayfeh $\mathrm{e}$ Mook, 1979).

O diagrama de estabilidade das soluções estáveis e instáveis podem ser representadas em um espaço de fase (kxג) chamado diagrama de Strutt (Figura 1).

Figura 1: Gráfico de $(k x \lambda)$.

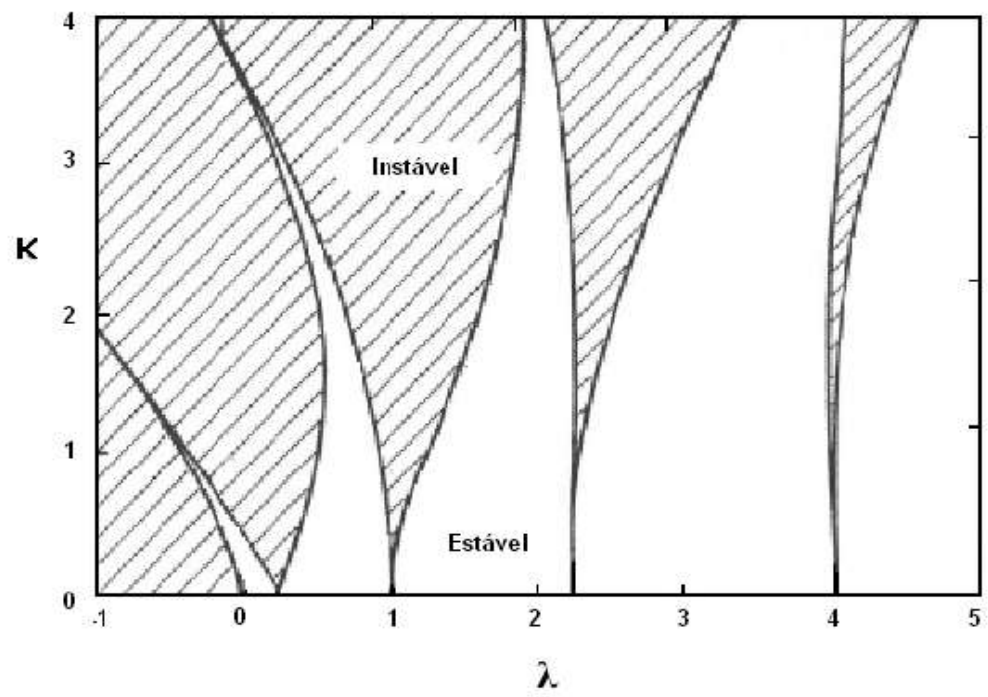

Fonte: Nayfeh e Mook (1979).

Na Figura 1 as regiões rachuradas representam soluções instáveis ou de ressonância, enquanto as não rachuradas são soluções estáveis. Os termos estável e instável referem-se ao espaço dos parâmetros $(k x \lambda)$, uma vez que $\mu$ está relacionado aos parâmetros $k, \lambda$, ou de maneira equivalente, atribuindo valores a $k, \lambda$, obtemos soluções instáveis ou estáveis da equação de Mathieu. O gráfico é simétrico em relação ao eixo $\lambda$. Verifica-se no o gráfico, (Figura 1), as regiões de estabilidade predominantes ocorrem para pequenos valores da constante $\mathrm{k}$. Por outro lado, as zonas de instabilidades que ocorrem de forma intercalada e decrescem para grandes valores da constante $\lambda$, principalmente devido ao amortecimento, que apesar de não estar sendo considerado, está presente em qualquer oscilador. Os efeitos do amortecimento contribuem para a redução das larguras das zonas de instabilidade (ressonância paramétricas). A equação (29) apresenta dois parâmetros governando o movimento, a saber; sua amplitude $k$ com que a frequência natural. É possível escolher valores para os parâmetros $\mathrm{k}$ e $\lambda$ de modo a fazer com que as bandas de instabilidade desapareçam. 
Consideremos a equação de (29), as constantes de separação $k \in \lambda$ se relacionam de acordo a equação, $k^{2}=4 g^{2} \lambda$. A Figura 2, mostra as regiões de estabilidades e instabilidades para o caso específico do movimento da partícula num campo magnético periódico paramétrico.

Figura 2: Gráfico de $(k x \lambda)$ para a partícula sob potencial tipo Mathieu.

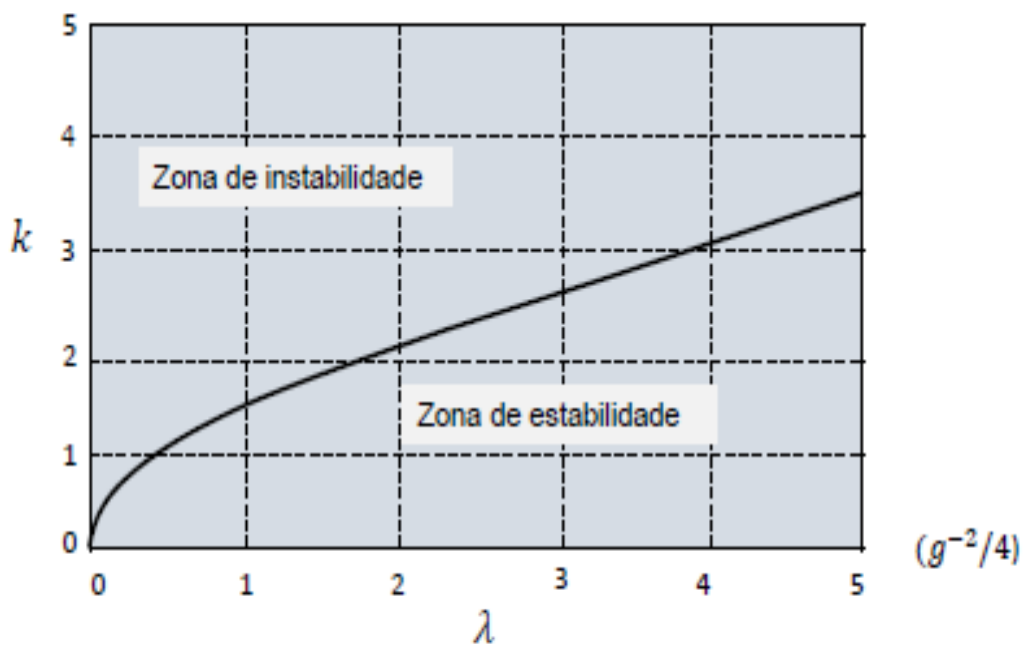

Fonte: Autores.

A sobreposição da Figura 1 na Figura 2, resulta no seguinte gráfico mostrado na Figura 3, abaixo.

Figura 3: Sobreposição das Figuras 1 e 2.

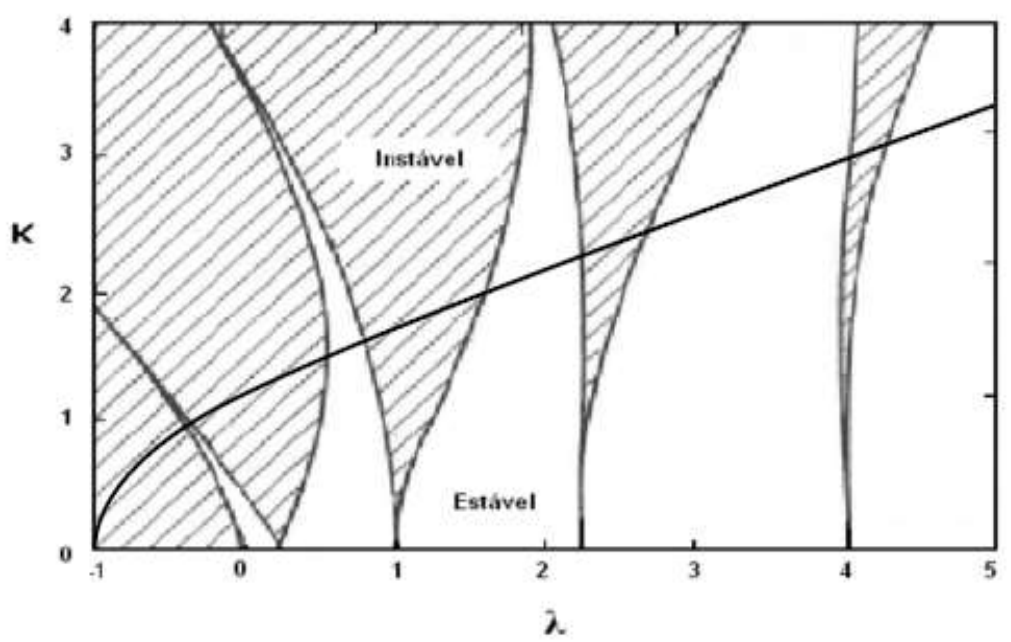

Fonte: Autores.

Observar-se na Figura 3 que apenas certos valores $\left(k_{n} ; \lambda_{n}\right)$ que satisfazem a condição de $k^{2}=4 g^{2} \lambda$ interceptam a curva dada pelo diagrama de Strutt dado pela Figura 1. Estes pontos fornecem as soluções permitidas para o problema da partícula num campo magnético paramétrico. Os pontos $\left(P_{n}, \lambda_{n}\right)$ que tornam a função $S(\phi)$ periódicas podem ser obtidos alternativamente resolvendo a equação (29) através do método de perturbação de autovalores (Nayfeh e Mook 1979). Esses pontos são dados pelas equações: 
$\lambda_{n}=\lambda_{n}^{0}\left[1+4 g^{2} I(\phi)\right]$

$k_{n}=2 \sqrt{g} \lambda_{n}^{0}\left[1+4 g^{2} I(\phi)\right]$

Onde:

$l(\phi)=\int_{0}^{2 \pi}\left|S_{n}^{0}(\phi)\right|^{2} \cos ^{2} \phi \cdot d \phi$

\section{A Discretização do Campo Magnético Externo}

Um resultado interessante obtido das soluções estáveis para o movimento oscilatório da partícula é o aparecimento de valores discretos para o campo magnético externo $\mathscr{H}_{0}$. Os valores que o campo magnético externo pode admitir no movimento são dados em função da equação (29), observando a equação (30), ou seja,

$\mathscr{H}_{\mathrm{n}}=\left[\frac{\omega \mathrm{c}}{\mathrm{m}}\right] \sqrt{\lambda_{\mathrm{n}}}$

Neste ponto, ressaltamos a importância da expressão (34), os campos magnéticos externos podem, em princípio, ser observados experimentalmente.

\section{Considerações Finais}

A transformação desacoplante (Bassalo, 1993) permitiu, modo original, um estudo detalhado de um dos diversos problema de Física Quântica relacionados a equação de Schrödinger de um sistema quadrático dependente do tempo para estados quânticos não-estacionários de um campo magnético com oscilação paramétrica. O estudo do movimento de uma partícula na presença de um campo magnético paramétrico evidência uma generalização do Problema de Landau (Landau, 1977). Com este estudo foi possível a observação de fatos interessantes, tais como: o aparecimento de uma periodicidade paramétrica na densidade de probabilidade $\rho(x, y, z, t)$ de encontrar a partícula numa dada região do espaço. Um outro resultado, de grande interesse teórico e experimental é a discretização do campo magnético externo $\mathcal{H}_{\mathrm{n}}\left(\lambda_{\mathrm{n}}\right)$ dado pela equação (35), a qual, caracteriza uma periodicidade nas soluções estáveis para a equação de Mathieu (28). Muitos destes resultados aqui apresentados encontram-se na dissertação de mestrado de Pampolha (1997).

Finalmente, conjectura-se o aparecimento de soluções caóticas para a região onde há instabilidade, como pode ser observado na Figura 2, as quais podem ser objeto de futuras pesquisas, assim como a generalização deste estudo para duas e três dimensões para o cálculo do propagador. 
Research, Society and Development, v. 10, n. 7, e16310716401, 2021

(CC BY 4.0) | ISSN 2525-3409 | DOI: http://dx.doi.org/10.33448/rsd-v10i7.16401

\section{Agradecimentos}

Todos os autores agradecem o apoio do IFPA, campus Belém e Santarém e da UFPA, Castanhal. Os autores C. R. Silva, J. P. da S. Alves e R. Germano agradecem ao MNPEF polo 37 UFPA e a SBF, enquanto que o autor D. P. M. Filho agradece ao MNPEF polo UFOPA, Santarém.

\section{Referências}

Abramowitz, M., \& Stegun, I. A. Stegun. (1965). Handbook of Mathematical Functions. Dover Publications.

Barros, V. P., Brtka, M., Gammal, A., \& Abdullaev, F. Kh. (2005). J. Phys. B: At. Mol. Opt. Phys. $38,4111$.

Bassalo, J. M. F. (1993). Nuovo Cimento 15, 28-33.

Demo, P. (2000). Metodologia do conhecimento científico. Atlas.

Filho, A. Ribeiro, \& Vasconcelos, D. S. (1994). Introdução ao Cálculo das Funções Elípticas Jacobianas. Ced.

Floquet, M. G. (1883). Sur les equations différentielles linéaires a coefficients périodiques. Ann. Ecole Norm. Sup. 12, 47-89.

Grib, A. A., Mamaev, S. G., \& Mostepanenko, V. M. (1994). Vacuum Quantum Effects in Strong Fields. Friedmann Laboratory Publishing.

Gutièrrez, J. C. et al. (2003). Mathieu functions, a visual approach. American Journal of Physics. 71, $233-242$.

Jesus, V. L. B., Guimarães, A. P., \& Oliveira, I. S. (1999). Classical and quantum mechanics of a charged particle in oscillating eletric and magnetic fields. Brazilian Journal of Physics 29.

Landau, L. D., \& Lifshitz, E. M. L. (1971). The Classical Theory of Fields. Mir.

Landau, L. D., \& Lifshitz, E. M. L. (1977). Quantum Mechanics: Non-relativistic Theory. Course of Theoretical Physics. Londres: Pergamon Press.

Magnus, W., \& S. Winkler. (1966). Hill's equation, Interscience tracts in pure and Applied Mathematics. Wiley-Interscience.

Mathieu, E. L. (1868). Le mouvement vibratoire dúne membrane de forma elliptique. J. de Math. Pures Appl. 13, $137-203$.

Nassar, A. B., Botelho, L. C. L., Bassalo, J. M. F., \& Alencar, P. T. S. (1990). Physica Scripta 42.

Nayfeh, A. H., \& Mook, D. T. (1979). Nonlinear oscilation. John Wiley and Sons.

Pampolha, J. B. S. (1997). Solução Exata da Equação de Schrödinger Dependente do Tempo via Transformação Espaço-Temporal. Dissertação de Mestrado em Física. Faculdade de Física, Universidade Federal do Pará.

Schmidt, A. G. M. (2019). Exact solutions of Schrödinger equation for a charged particle on a sphere and on a cylinder in uniform eletric and magnetic fields. Physics E 106, 200-207.

Schmidt, A. G. M. (2020). Exact solutions to Schrödinger equation for a charged particle on a torus in uniform eletric and magnetic fields. Brazilian Journal of Physics 50, 419-429.

Silva, C. da R., Pampolha, J. B. S., Alves, J. P. da S., Germano, R., \& Júnior, W. P. (2020). Experimento de Física de baixo custo para o Ensino Médio: Estimando a resistividade elétrica de tubos de metais não-ferromagnéticos utilizando ímãs de neodímio e um cronômetro. Research, Society and Development.

Sudiarta, I. W., \& Geldart, D. J. W. (2008). Solving the Schrödinger equation for a charged particle in a magnetic field using the finite difference time domain method. Phys. Lett. A 18, 3145.

Tannoudji, C. C., Diu, B., \& Laloe, F. (2006). Quantum Mechanics. Wiley-Interscience.

Wentzel, G. (1981). Z. Phys. 22, 518.

Zanchin, V., Maia, A., Craig, W., \& Brandenberger, R. (1998). Phys. Rev. D 57, 4651. 